SOI: $1.1 /$ TAS DOI: $10.15863 /$ TAS

International Scientific Journal Theoretical \& Applied Science

p-ISSN: 2308-4944 (print)

e-ISSN: 2409-0085 (online)

Year: 2015

Issue: 08

Volume: 28

Published: 30.08 .2015

http://T-Science.org

SECTION 12. Geology. Anthropology.

Archaeology.
Elnur Latif oglu Hasanov Corresponding member of International Academy of Theoretical and Applied Sciences, Ph.D., leading (senior) specialist of Ganja Department Azerbaijan National Academy of Sciences,

Ganja, Azerbaijan 1-hasan@hotmail.com

Nicolash Eugster Ph.D., University of Fribourg Boulevard de Pérolles, Fribourg, Switzerland

\title{
INNOVATIVE ARGUMENTS ON RESEARCH OF HERITAGE OF M. SHAFI ON THE BASIS OF ETHNO-HISTORIC AND LITERARY SOURCES
}

\author{
Abstract: Basic features of creation of the famous philosopher and poet of Azerbaijan M. Vazeh were \\ researched on the basis of historic-ethnographic and literary arguments by scientists from Switzerland and \\ Azerbaijan. \\ Key words: Azerbaijan, Ganja, XIX century, innovative research methods, culturology, historic-ethnographic \\ investigation. \\ Language: French \\ Citation: Hasanov EL, Eugster N (2015) INNOVATIVE ARGUMENTS ON RESEARCH OF HERITAGE \\ OF M. SHAFI ON THE BASIS OF ETHNO-HISTORIC AND LITERARY SOURCES. ISJ Theoretical \& \\ Applied Science 08 (28): 32-36. \\ Soi: http://s-o-i.org/1.1/TAS-08-28-4 Doi: crossef http://dx.doi.org/10.15863/TAS.2015.08.28.4

\section{ARGUMENTS INNOVANTES POUR LA RECHERCHE DU PATRIMOINE DE M. CHAFI SUR LA BASE DES SOURCES ETHNO-HISTORIQUES ET LITTÉRAIRES}

Abstract: Les caractéristiques de base de la création du célèbre philosophe et poète d'Azerbaïdjan M. Vazeh ont été étudiés sur la base d'arguments historiques-ethnographiques et littéraires par des scientifiques de la Suisse et de l'Azerbaïdjan.

Mots-clés: Azerbaïdjan, Gandja, XIX e siècle, les méthodes de recherche innovantes, culturologie, enquête historique et ethnographique.

\section{Introduction}

La situation climatique importante (de la région), le beau paysage, la fortune naturelle riche, les resources d'homme de la région font connaître Gandja non seulement en Azerbaïdjan, mais aussi dans le proche et moyen Orient comme un des centres culturels, économiques et industriels. Malgré le changement de l'emplacement, le château est toujours appelé par la population locale. Il s'agit d'une très vaste zone de forêt et dans le forêt, avec les matériaux de construction locaux construite sur les ruines de la magnifique château et les murs de la ville et les ravitaillement du port atteint jusqu'à nos jours. Un géographe éminent Strabon qui vivait au premier siècle avant J.C. avait écrit sur les condition géographiques naturelles de Gandja. La terre planté une fois donne la fruit une fois, même deux fois cinquant fois plus an, dans la condition sans mettre au pacifique, la bourer avec l'araire. Toute les plaine étaient arrosées avec les rivières et d'autres eaux mieux qu'en Egypte et Babilon. C'est pourquoi il y avait beaucoup de pâturages. En outre, ici l'air était pur que là bas. Strabon avait comparé le territoire de Gandja avec celui de Babilon et l'Egypte qui avait 5 mille ans de culture. La terre de Gandja était plus préfére que celui de l'Egypte et Babilon. Depuis sa création et l'emplacement d'au moins 5 fois, l'évolution des aspects géopolitiques et stratégiques situait dans une situation favorable. Gandja attirait toujours l'attention des envaisseurs.

Plusieurs fois Gandja étant la victime de quelques tremblements de terre, ainsi que les Perses, les Géoggiens, Kharazm, les Mongoles, les Arabes et les russes ont attaqué sur Gandja et l'ont ruiné complètement.

Dans l'arène de la guerre et de la destruction causée par des coupes répétés à Gandja, la ville n'est pas tremblé. Gandja s'est devoloppé en prenant la force des racines turque au niveau des grandes villes. 
Et pendent l'époque de moyen âge, il y avait un système de transport excellent de Gandja. Si en Europe le moyen de transport souterain était construit au milieu de $\mathrm{XX}^{\mathrm{e}}$ siécles. Au moyen âge, à Gandja avait été déjà construit un système de route souterrain à deux étages qui pourrait traverser les phaétons. Ici il y avait la circulation du peuple et de transport, en même temps on y avait situé les autres systèmes de communication.

C'est pourqoui les vers de Mirzé Chafi Vazeh était respectés en étranger dans cette époque - là et ceux - ci fait connaître leur auteur. A propos ses oeuvres ne sont pas gardés en Azerbaïdjan, il n'a pas été connu dans son pays natal. L'un des premiers éducateurs du XIX siècle Mirzé Chafi était contemporain de Abbasqoulou Aga Bakikhanov et dévancier de Mirze Fatali Akhundov. La poésie lyrique et philosophique avait été connue en Europe et en Russie. Ses vers rappelaient aux lecteurs europeens et aux lecteurs russes le grand poète persan Eumar Khaïam.

Mirzé Chafi Vazeh est né en 1794 à Gandja dans une famille d'un maçon qui s'appelait Karbalai Sadiq. Il s'occupait de travaux architecturaux dans le palais du Khan de Gandja Djavad Khan.

Mais Chafi a perdu son père quand il était très jeune. Il a vécu sous les auspices de son parent Hadji Abdulla. Son fils devient un clergé. Chafi a commencé à faire ses études réligieuses. Mais comme il s'intéressait à l'enseignement laïque, dans le medrécé (une école réligieuse) les clergés ont refusé de lui enseigner. En fait. Mirze Chafi Vazeh a dû quitter le medrécé. Sur cet événement orientimaliste Adolf Berjé a écrit dans ses mémoires. Après avoir quitté le medrécé il a commencé à s'instruire à developper ses connaissances intellectuelles et il a apris la langue persanne. Ça lui a permis de connaitre les grands poètes de l'Orient et ses oeuvres.

A l'aide de Hadji Abdoulla Mirze Chafi Vazeh dirigeait les travails du palais de Pusté Khanoum, la fille de Djavad Khan.

En 1826 pendant la guerre entre la Russie et l'Iran Pusté Khanoum est oubligée de s'en fuire à l'Iran avec son frère Ougourlou Khan. Mirza Chafi reste sans travail, en ce moment-là Hadji Abdoulla meurt.

Malgré que Mirzé Chafi reste seul, il ne tombe pas dans la déprétion, il commence à enseigner aux enfants et s'occupe d'apprendre l'écriture aux enfants. Un de ces enfants était Mirzé Fétéli Akhoundov qui apprenait l'écriture.

En 1840 Mirza Chafi Vazeh arrive de Candja à Tiflis. Au mois de novembre de la même année, à l'aide de Mirza Fatali Akhundov il est nommé le professeur d' azerbaijanais et persan à l'école de «Qəza» (territoire) à Tiflis. Il habite à Tiflis jusqu 'a la fin de l'année 1846. Après la sépartion de Tiflis il a écrit la poésie «Adieu Tiflis» en langue persanne. En 1844 il crée une colloque pilosophique, littéraire «Divani-hikmat» à Tiflis. Dans la soicée «Divani Hikmat» on lisait avant des vers et puis on faisait des dicussions.

\section{Materials and methods}

A ce temps là, dans le colloque de vers participaient Abbaskoulau aga Bakikhanov. M.F.Akhoundov, les poètes Najami, Cheula, Mirza Hassan, Hadji Abdulla, Mirza Yousif, Vidadi, Haci Yusif. Grâce à l'activité de cette colloque Mirza Chafi Vazeh à fait la connaissace avec les hommes de science célèbres et hommes de profession. (mitier)

L'un des membres de cette colloque était Fridrikh Fon Bodenchtedt né en 1819, au Province Payné se trouvant à côté de Honnover, la captale de Bas Sakson de'Allemagne. Bodenchtedtétait juif. Il avait fait ses études dans les universités célèbres comme Guétinguène, Munhène, Berlin.

En 1841, Bodenchtetd arrive à Moscou et la il s'occupe de l'éducation des enfants de knyaz Mikhail Qolitsin.

En 1844, avec l'inivitation de générale Neydtqart, juge en tête de caucase, il arrive à Tiflis et y travaille en qualité de professeur. Il apprenait la langue persanne. Mirza Chafi a son tour, faisait la connaissance avec les oeurvres des classiques de 1 'europe d'ouest, a l'aide de lui.

En 1846 Bodenchtedt rentre en Allemagne. Dans les années 1889- 1890 il va aux Etats - Unis. En 1892 il est mort et il est enterré dans la ville Visbaden de l'Allemagne.

En 1846, Mirza Chafi rentre à Gandja. Il est nommé en comme le professeur à l'école «Qəza» (territoire) ouverte par l'état Attenant à professeurat, il s'occupe aussi de la créativité de poésie.

En 1850, au mois de janvier il rentre à Tiflis et commence à travailler en qualité de professeur de la langue azerbaidjanaise à la gymnase des nobles. Il y habite jusqu à la mort (à la fin de sa vie).

En 1852, le 28 novembre, il est derrière du jardin Botanique.

Aujourd'hui, il n'y a pas d'originaire en persan et azerbaïdjanais édité en russe et dans les langues de l'europe d'ouest.

Les manuscrits des vers sont emportés en Allemangne par Fridrix fon Boudenchtedt.

Après la rencontre avec Bodenstedt à Tiflis en 1844, Mirzé Chéfi faisait écrire ses poésies. Outre cela Mirzé Chéfi a offert à Bodenstedt son recueil de poésies en manuscrit.Bodenstedt, lui-même écrivait: «Il m'a offert un cahier nommé «La clef des esprits». Dans l'avant-propos de ce cahier, Mirzé Chéfi,lui,écrivait: «Moi, Mirzé Chéfi, je lui offre mon «medjmoueyi-assari» (recueil de poésies) qui consistent en soi des guéssidés (odes), des guazelles, des murébbéates et des messnévis (des distiques). Ayant vécu (été) à Tifflis, F.Bodenstedt ramasse les poésies de Mirzé Chéfi et 
en 1846, il les emporte en Allemagne et après avoir traduit, il les fait publier. Une partie de ces poésies ont étéintroduites dans son oeuvre «Mille et un jour à l'Est ».

Après son oeuvre,en 1851, F. Bodenstedt fait publier à Berlin un petit livret nommé «Les chants de Mirzé Chéfi». Celui-ci, après une diffusion rapide, rend son auteur très célèbre. Peu après les oeuvres de Mirzé Chéfi se répandent vite dans toute l'Europe Occidentale. Outre l'allemand, elles sont traduites en anglais, français, italien, suisse, norvégien, holland, danois, polognais, suédois, tchèque et même en ivrite (juive). En Russsie aussi,ces poésies éveillent un grand intérêt. La traduction russe des «chants» et son édition appartient à l'ami de N.G.Tchernichevski, poète Mikhail Larinovitch Mikhailov (1826-1865). Après lui, les poésies de Mirzé Chéfi ont été traduites par V.M.Markov, M.Ramch, N.Eyfert, et les autres. Ayant pris les connaissances de ces poésies L.M.Tolstoy les avait bien appréciées. Les poésies de Mirzé Chéfi ont été publiées six fois en 1868, douze fois en 1876. En somme elles ont été publiées 169 fois jusqu'à 1922. Aucun auteur ne connait un tel succès de publication.

Ayant remarqué ce grand succès, en 1873, Bodenstedt trahit à son maître en tâchant de se réclamer le vrai auteur. Orientaliste Adolf Berger en s'appuyant sur les paroles du cheikh-ul-islam deCaucase, mollah Ahmad Salyani, nie absolument le talent de poète et le droit d'auteur de Mirzé Chéfi. en trompant la société littéraire Bodenstedt a dit qu'il avait d'abord édité ses oeuvres sous le nom Mirzé Chéfi, sous l'impression de son voyage à l'Est...

Comme s`il a pris le nom de Mirza Shafi comme un pseudonyme oriental.Dans les années suivantes Bodenshtedt a pu reunir des admirateurs autour de soi, alors que pendant ce temps Vazeh a été oublié par le public. Quoiqu`il joue un rôle important dans la diffusion des poemes de Vazeh en Europe, il était aussi marqué comme un imitateur de sorte que ca lui a apporté le déshonneur. Il a fait une grande popularité en publiant les poèmes de Vazeh d'une manière privée ,mais il a aussi gagné lincroyance du public en faisant oublier le nom de Vazeh.

Il est a noter que Bodenshteidtedt a ajouté a ce recueil non seulment les poèmes de Vazeh il a ajouté aussi ceux qui ne lui appartenaient pas.

Malgré la disparition des poèmes de Vazeh en Allemagne,certaines poèmes ont étè trouvé par les chercheurs. Les poèmes trouvés consistent d`une qazelle, un pentametre, un qita et trois couplet en azéri,trois qazelle et deux vers en perse.

Le début du gazelle commence par les rimes suivants:

Puisqu'il ya des corps célectes dans le ciel.
Jaai autant de blessure de côquette sur ma poitrine.

Parfois on comptait l'auteur de ce poème comme Molla Veli Vidadi en s`appuyant aux fausses sources. Ces poèmes avaient été découvertes pour la première fois par Sultan Mumtaz. Pour étudier les oeuvres de Vazeh profondement et obtenir des résultats concrets, il faut les rechercher en azéri et en perse des sources originaux non pas à travers des traductions. Dans ce recueil il ya le poème de Zuleykha, Mirza Yusif, les chansons d'amour,de consolation, de la rose etc. Quoique Vazeh accepte le poète Perse Hafiz comme son maitre, dans ses oeuvres on peut sentir l' esprit, l' âme et la génie des oeuvres de Fuzuli. Pour cela, il faut apprécier Vazeh comme successeur de Fuzuli dans le monde littéraire.

Dans ces modèles on critiquait les religieux qui sont loin de la verité. Pour ça les réligieux ne l'aimaient pas. Le contemporain de M. Chafi, Akhound Molla Husein Pichnamazadeh qui était clergé de Gandja l'appellait perfide. Les réligieux ne l'aimait guerre car dans ses poèsies $\mathrm{M}$. Chafi utilisait toujours les mots pinards, bien aimées, amante, maitresse etc.

Les réligieux ne comprenaient pas que ces mots étaient des exemplaires littérraires. Dans les mêmes mots M. Chafi chantait l'amour extraordinaire plus forte que l'amour mondaine. C'est une pureté moral. Dès 1840 M. Chafis occupait de la langue azerbaidjanaise dans le lycée ruse. Dans les lycées ouverts aux pays du Caucas on étudiait en azéri et en pers. C'est pourqoui on avait besoin les manuels écrits dans ces langues. Pour la langue pers anne on utilisait l'oeuvre d'Abbaskulu aga Bakikhanov «Qanuni-Qudsi » (qui présente les régles de la grammaire du pers). Pour instruction la langue azerbaidjanaisesil n'y avait aucun manuel. C'est pourquoi M. Chafi et l' étudiant de Mirze Kazim bek la prof. de pers Ivan Qriqoriev form une chrestomathie consernant la lange Azerbaidjanais, le dictionnaire de cette chrestomathie était prête en 1851. La chrestomathie était prête au mois d'avril. Cette chrestomathie s'appelait «Tatarskaya krestomatiya Azerbaydjanskoqo nareçiya».

Cette chrestomathie consistait deux parties: première partie s'appelait «Polnaya ili prostrannaya xrestomatiya azerbaydjanskoqo nareçiya» deuxième partie «Izvleçeniya iz prostrannoy xrestomatiya». Dans certaines sources ondisait le manuel préparé par Mirzé Chafi à la collaboration avec le prof. Chargé de cours de lycée le prof. Tifflisse Ivan Qriqoriyev nommait «Kitabi-türki», «Chrestomathie du dialecte azerbaidjanais préparé par Qriqoriyev et Mirza Chafi Sadiq oglu».

Le premier de ces livres a été redigé pour les élèves supérieurs du lycée et le deuxième était pour les débutant et pour des écoles de province. Deuxième volume a été paru par Ivan Grigoriev a Tabriz en 1855 après la mort de Mirzé Chafi Vazeh 
et celui-ci fut utilisé, comme un manuel pour apprendre l'azerbaidjanais au lycée et dans les écoles de province. L'édition complète de ce livre apparut à la fin de l'année 1856 et a envoyé au Ministère de l'Education pour la permission de son utilisation comme un manuel. Mais certains proffesseurs de l'Université de Peterbourg ont écrit des opinions négatives.

Outres les dictionnaires, ce manuel se divise en trois parties. La première partie se compose des articles traduits des langues arabe et personne en azerbaidjanais. La plupart de ces articles sont des fables et des préceptes de morales. Dans cette partie il y a de brèves informations sur l'histoire antique de l'orient jusqu'à l'Islam qui est prise de l'oeuvre «Reuvzetussefa» de Mirkhounde.

\section{Conclusion and Recommendations}

Dans la deuxième partie on présente des aforizmes et des citations citées de différents auteurs. Elles étaient traduites comme oeuvres «Garabakhnamé» et «Derbendnamé» / Dans la troisième partie on présente beaucoup de vers. Les guéssidés et les extraits du poème «Leyli et Medjnoun»y sont introduits aussi. Ainsi, on peut dire que Mirzé Chafi Vazeh a présenté le premier manuel en azerbaidjanais. Après ce manuel à la deuxième cinquantaine du XIX siècle Mirzé Abdulhassan Vézirov, Seyyid Azim Chirvani, Rachid bek Efendiyev ont continué cette tradition-d'écrire les manuels pour les écoles.

On a fait beaucoup de recherches sur la vie et sur l'activité de Mirzé Chafi Vazeh dans son pays natal et dans des autres pays. En XIX siècle l'orientaliste le Almande Adolf Bergé a fait imprimer un guézel écrit en persanne un tekbeyt (c'est une espèce d'un vers de la poèsie oriantale) de Mirze Chafi Vazeh et la traduction de A. F. Veltmande dans la revue de l'Association Orientalisme d'Allemande.

$\mathrm{Au}$ début de $\mathrm{XX}^{\mathrm{e}}$ sicèle, A Gandja, Salman Mumtaz Asguérov a ramassé les manuscrits des poètes locals et il a découvert 8 vers appartenant à Mirza Chafi préparés par Mirza Mehdi. Mais avant de ça Salman Mumtaz fait imprimé quelques couplets de Mirza Chéfi dans le journal "Qourtoulouche" (1920, №1) .

En 1926 Salman Mumtaz a fait imprimé les vers trouvés de Mirza Chafi dans le journal "Rommaunisse" sous le nom de livre "Mirza Chafi".

Aprés Mumtaz le professeur Aliajdar Saidzade (1899-1970) s'occupe de l'héritage de "Mirza Chafi".

En 1929 Aliajdar Saidzada à écrit livre "Le célèbre penseur et poète Mirza Chafi Gandjanais" à l'occasion de journée de "Mirza Chafi" organisée à Gandja.

Le livre a été imprimé dans les langues russe et azerbaïdjanais.
En 1961 on a imprimé en azerbaïdjanais le livre les chants de "Mirza Chafi".

On a fait la recherche sur Mirza Chafi Vazeh en Russie, en Europe et dans d'autres pays. On avait imprimé ses oeuvres en les traduisant. Les gens cultivés qui s'intéressaient à la festin «Divani Hikmet» qu'il avait organisé, ont esayé reunir ses manuscrits. L'un d'eux était Ivan Konstantinoviç Yenipopov. Il a réuni beaucoup de matérieux de Mirza Chéfi Vazeh.

Hélas on n'avait pas trouvé ces matériaux. Les oeuvres de Mirza Chafi Vazeh avaient été traduits en géorgien dans les années $60 \mathrm{du}$ XIX siècle. Certains de ces traductions avaientt été imprimés dans le journal «Iveriya»sous la rédaction de Ilya Tchavtchavadzé. 114 poèsies de Mirza Chéfi Vazeh ont été traduites en géorgien par Akani Gelovani. Proffesseur Aftandil Savadzé a traduit ses deux poésies. Encore a Tbilissi l'imprimérie «Art»a édité le livre de Akaki Gelovanini qui s'appelle «La symphonie de safes» consacré à Mirza Chafi Vazeh».

En Estonie, Johan Liyv a traduit deux vers de Mirza Chafi Vazeh en estonien.

En Allemagne on a fait un grand travail de recherche sur la personnalité et la créativité de Mirza Chafi. En 1889 en Allemagne a paru le livre du polyglotte lituanien Yourguisse Youliousse Laverveynasse intitulé Divan (Le recueil des guézelles) occidental et oriental de Mirza Chafi. Le chercheur K.Sundermayer a redigé une thèse nommée Les chants de Frirdrikh Fon Bodainshtedt et de Mirza Shafi.

Y. Moundelhenk a publié en 1978 à Ha, bourgue le livre "Fridrikh Bodainshtedt et Mirza Chafidans la critique littéraire azerbaïdjanaise". Après cela beaucoup d'articles sur Mirza Chafi ont été publié dans la presse allemande. Dans certains oeuvres Mirza Shafi est reconnu comme grand poète et penseur azerbaïdjanais. En 1967 à Kologne, Yssa Kéhabi originaire de Perse a soutenu une thèse sur le sujet "L'allemagnisation des chants de Hafiz par Fridrikh Bodainshtedt".

D'après l'auteur Mirza Chafi n'était pas poète. Son métier n'était que calligraphe. Mais il oublie qu'au XIXe siècle les azerbaïdjanais étaient reconnus comme turc et tatar. Pour cacher cette vérité il prétend qu'à Tbilisi, (la ville Caucasienne), était impossible l'apprentissage de la langue tatare. Malgré que la tatar était l'une des langues turques il n'était pas la langue parlée au Caucase.

C'est pourquoi on n'a pas besoin de l'apprendre à l'école secondaire dans les autres endroits de la Russie. A cause de son antipathie envers les Azerbaidjanais, qu'il crée exprès de fausse imagination dans l'esprit occidentale consernant les noms Azerbaidjanais, Tatars et Turcs. Quoiqu'il nomme sincèrement, avec modestie Hafiz comme son maître, on peut sentir l'esprit de Fuzouli dans sa créativité. Tant Hafiz est grand en Perse, autant il est 


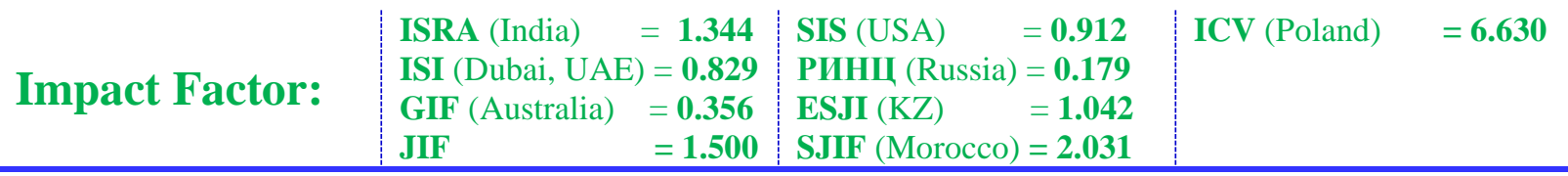

petit devant la génie de Fuzouli. Les recherches essentielles consernant la riche créativité de Mirza Chafi seront entamées en avenir.

L'ordre du président de la république datée de 3 février 2014 consernant la célébration de 220 e anniversaire de Mirza Chafi Vazeh dresse une grande obligation devant les chercheurs de Mirza Shafi.
Comme Nizami, Hassanoglu, Nassimi, Fuzouli et d'autres génies, Mirza Chafi Vazeh, lui aussi, est une étoile brillante $\mathrm{du}$ monde spirituel $\mathrm{du}$ peuple Azerbaidjanais. Sa vie et sa créativité sont un exemple pour notre histoire culturelle et un modèle pour notre monde de poésie.

\section{References:}

1. Aslanov A (2004) M.Ş.Vazeh. Nəğmələr. Bakı: Şərq-Qərb.

2. Bayramov A (2008) Fridrix Bodenştedtin Mirzo Şəfi Vazeh haqqında xatirələri. Bakı, Nurlan, $108 \mathrm{p}$.

3. Guliyeva NM, Häsänov EL (2014) Die traditionelle Gändschänischen Teppiche von Zeitraum der Aserbaidschanischen Gelehrten und Dichter Mirsä Schäfi Waseh als ethnoanthropologische quelle (XIX Jahrhundert). European Applied Sciences, 2, pp. 3-5.

4. Həvilov HA (1991) Azərbaycan etnoqrafiyas1. Bakı: Elm

5. Osgərli Z (2010) Mirzə Şəfi Vazeh. Bak1, Nurlan, 92 p.
6. Mustafaev FA (1972) Ya k svetu shol. Baku, $197 \mathrm{p}$.

7. Oliyeva NU (2013) Mirzə Şəfi Şərq-Qərb araşdırmalarında. Gəncə, Elm.

8. Smith WB, Hasanov EL (2013) Importance of handicraft traditions in investigation of history of urban culture in Ganja. ISJ Theoretical \& Applied Science. 11(7): 61-66. doi: http://dx.doi.org/10.15863/TAS.2013.11.7.10

9. Tərlanov M, Ofəndiyev R (1960) Azərbaycan xalq sənəti. Bakı: Uşaq gənc nəşr.

10. Veysəlli F (2010) Mirzə Şəfi Vazeh və Fridrix fon Bodenştedt: yozmalar və faktlar. Bakı, Təhsil, $156 \mathrm{p}$.

Elnur Latif Hasanov

membre correspondant del'Académie internationale des sciences théoriques et appliquées, Ph.D. étudiant de troisième cycle spécialiste de la Direction générale de Gandja de l'Académie Nationale des Sciences d'Azerbaïdjan,

Gandja, Azerbaïdjan

1-hasan@hotmail.com

Nicolash Eugster,

Ph.D., Université de Fribourg Boulevard de Pérolles, Fribourg, Suisse, (Fribourg, Suisse) 